should be held at an early date to formulate such a scheme. Even if to-morrow the whole of the available chemical force set to work on some organised plan, it would not be any too soon to get the necessary information together for the use of the existing works and the new works when they are started.

The urgency of this action is further shown by a resolution passed by an important meeting held in Manchester on February 16 , which was presided over by Sir Chas. Macara. The resolution, which was carried unanimously, stated: "That in the opinion of this meeting the Government would do well to organise immediately the present chemical talent in the country with a view to chemical research being undertaken for, and on behalf of, all manufacturers interested, and that the services of these experts should be available for all desirous of availing themselves thereof."

The adoption of such a plan for bringing the educational institutions more closely into touch with the industries would in all probability mark the beginning of a new era in which both would benefit. The more intimate association of professors of chemistry with chemical industry would introduce into the works that higher ideal and broader scientific spirit upon which successful research and development depend, whilst the schools would benefit by the great incentive of practical reality.

\section{The Parliamentary Debate.}

Since the above was written, the important debate in the House of Commons on Monday, February 22 , has advanced the matter another stage. The criticisms offered by various speakers raised few novel points, and the general tone of the debate was, on the whole, favourable to the Government scheme. Several speakers, including Sir Philip Magnus, expressed the opinion that the grant of Ioo,oool. for research work was totally inadequate. Mr. A. Chamberlain and others emphasised the great difficulties the undertaking would have to face on the conclusion of the war, and many speakers acknowledged that the question of the manufacture of dyes was of such a special character as to warrant quite exceptional treatment.

Mr. Runciman, speaking on behalf of the Government, made the interesting announcement that upwards of $400,000 l$. has already been subscribed towards the capital of the new company, and further stated that the signing of the five years' agreement was not an essential condition of subscribing capital, but that priority of supply would be given to those who had given the undertaking. His concluding remarks may be quoted : "I am surprised that anyone should suggest that we have not adequate chemical knowledge in this country. We have sufficient first-class men, but we lack an adequate number of second-class chemists, which we shall not produce if they are only paid $3 l$. a week.

"Even if this were only an emergency scheme the million pounds promised by the Government

NO. 2365 , VOL. 94] would be well spent. A single month of unemployment in the textile trade would mop up more than that of our national money. It is the disadvantage of all practical schemes that they are full of compromises, but no step has been taken without a practical reason."

WALter M. Gardner.

\section{ON COLOUR SENSITISED PLATES.}

\section{II.-Their Applications. Panchromatic Plates. Testing Dyes, etc.}

The commercial "ortho-" or "iso-chromatic" plates, which are specially sensitised for green, besides their obvious uses for scientific purposes in which red sensitiveness is not necessary, are of especial use in the photography of coloured objects where an improved result, as compared with an ordinary plate, is desired, rather than a full correction. As the unassisted eye is not a very keen critic of the relative brightnesses of different colours, especially when they are not in juxtaposition, such an improvement is often all that is necessary. The deficient sensitiveness to red causes a pure red to be represented as if it were black. But almost all colours in nature and most artificial colours are not pure, and so far as a red contained any green or white, that is so far as it reflected any other light whatever in addition to the pure red, except pure blue, then the rendering of the red would be improved. But this fact as to most natural colours being mixed is also a disadvantage, for all coloured objects, so far as they reflect any red, will suffer because of the want of red sensitiveness of these plates.

Plates are made specially sensitised to red and called red and yellow sensitive, and practically all that has been said with regard to green sensitised plates, both now and in the previous article, applies to these, only that the deficiency here is green instead of red.

By the use of more than one sensitiser, plates may be sensitised to both green and red, and the early panchromatic plates were of this type. They had three maxima of sensitiveness, one in the blue due to the plate (that is, the silver bromide) not specially sensitised, and one in the green and one in the red due to the two sensitisers. Such plates may be regarded as being as much of an improvement on "orthochromatic" plates as these are on ordinary plates, but the sensitiveness is uneven, and they consequently suffer as already described. By the careful selection and combination of sensitisers this unevenness has been very largely overcome in the panchromatic plates of the present day. Excessive blue sensitiveness of course remains, but this is easily obviated by a pure yellow filter.

The three illustrations (Fig. 2, $a, b, c$ ) show the effects of various colours by the use of ordinary, orthochromatic, and panchromatic plates, respectively. The colour filter used with the orthochromatic plate might with advantage have been 1 Continued from p. 677 . 
rather darker. The " $\mathrm{L}$ " near the middle is deep blue, the word "mark" is white, and the ground they are on is yellow. The book covers are red. It will be noticed how the panchromatic plate gives the yellows better than the orthochromatic plate,

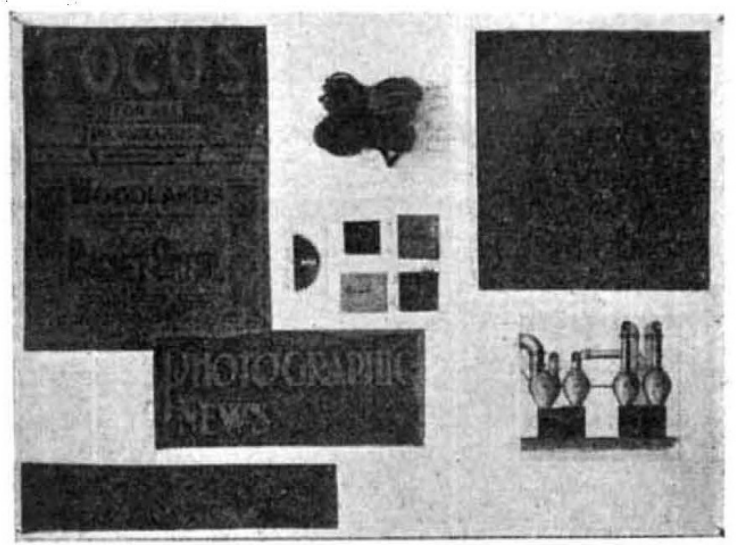

(a) Ordinaty Plate.

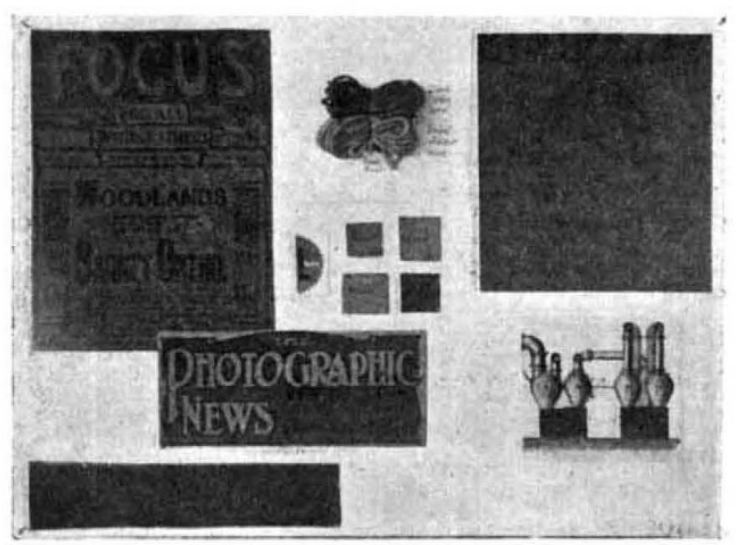

(b) Isochromatic Plate and Yellow $\left(x_{4}\right)$ screen.

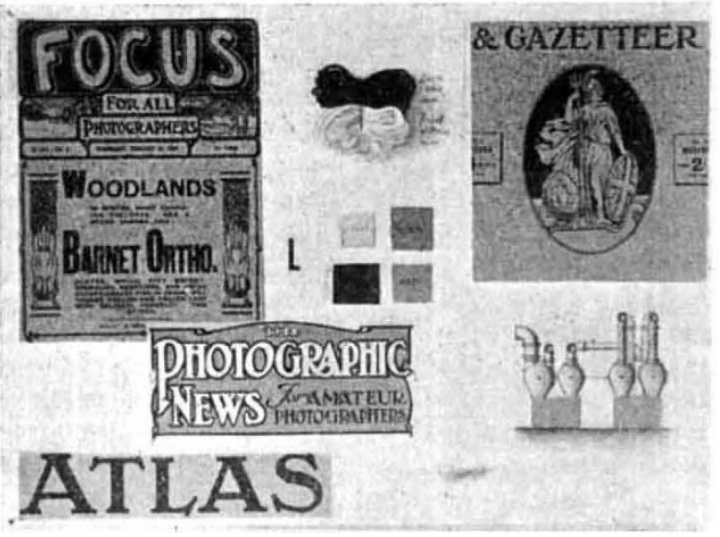

(c) Panchromatic Plate and Yellow $\left(\mathrm{K}_{3}\right)$ Screen.

$$
\text { FIG. } 2 \text {. }
$$

and gives the reds, which the orthochromatic plate renders in the "Gazetteer" and "Atlas" as if the red paper was of the same colour as the black printing ink. Other effects will be clear without further description.

$$
\text { No. } 2365 \text {, VOL. } 94]
$$

Panchromatic plates have been made commercially for fifteen years or more, but the plates of the last few years are very superior to their predecessors. Some makers issue more than one kind, the variation so far as colour sensitiveness is concerned being chiefly as to the extent of the red sensitiveness. Some are sensitive only to about the red lithium line or the $\mathrm{C}$ line in the solar spectrum. Others are sensitive to beyond the A line or the red potassium lines, and plates can be supplied that are sensitive considerably further into the infra-red. For the correct representation of coloured objects infra-red, and even the extreme red, sensitiveness is' only harmful, just as ultra-violet sensitiveness is, because these radiations are dark to the eye. It is well to choose a plate for this purpose that is not sensitised to the extreme red, and it is necessary to use a colour filter that cuts off the ultra-violet (some of the older yellow filters did not do this) and reduces the intensity of the blue so that it does not produce an undue effect. With a modern panchromatic plate a suitable filter will require the exposure to be increased perhaps only five times, as compared with the exposure necessary for a white object without the filter. This does not mean that such a plate and filter will give a sensitiveness curve that will exactly coincide with the luminosity curve of the solar spectrum, but it is a very near approach to it, so near that for general purposes it is more important to see that the present degree of perfection is maintained, rather than that it is increased, though of course we hope for still better results in due time.

There are many uses to which colour sensitised plates and colour filters may be put besides the correct representation of colour luminosities in the photography of objects, spectrum photography, and in connection with three-colour reproduction processes. Given black and white and any single colour, it is possible to photograph the three so that the colour is represented as if it were either black or white. Take the simple case of a drawing or manuscript in black ink on white paper with alterations in red ink. The red alterations can be made to appear as black as the black ink by using blue (or blue and' green) light only, that is, a light which has no red in it. An ordinary plate, which is sensitive only to blue, will give this result. But the red alterations can be made to appear as white, and therefore to disappear, by using red light only, that is, a plate sensitised to red in conjunction with a red colour filter. Engineers' drawings in blue, as when copied by the ferroprussiate process, give poor contrast on ordinary plates because the active light is chiefly blue, but with a red sensitised plate and red (or yellow) filter the blue becomes practically black. Similarly it is often possible in photography to eliminate stains on documents, or to find an inscription that has faded into invisibility. In this latter case it may be well to use extreme measures, as including as much ultra-violet as possible in one experiment, and the 
extreme red in another, if the colour character of the detail being searched for is unknown.

By modifying such extreme measures as just described, it is obviously possible by photography to intensify or diminish the apparent effect of any colour, and so, as for example in stained physiological sections, to improve on the original, or from one original to get two (or more) photographs that represent the section in, as it were, two (or more) different conditions as to staining.

The photographer has also by such means a very large measure of control over the effect of mist. The writer has shown that light is scattered by particles of a diameter equal to half a wavelength of the scattered light. By passing a brilliant pencil of light through a flask of filtered distilled water the path of the pencil may be scarcely visible (the "Tyndall effect"). But by photographing the arrangement on an ordinary plate, that is, using blue and ultra-violet light, the path of the light will probably be shown very conspicuously indeed, the particles that escaped removal by filtration being not large enough to scatter light of greater wave-length than the ultra-violet and the extreme blue. When the particles of the mist or turbidity are graduated in size, as they generally are, and are not coarse like the dust that may be stirred up from a dry road, then the longer the wave-length of the light used the less does the mistiness show in the photograph. When the air is misty, by the use of a yellow filter distant objects may be photographed more clearly than they can be seen, and by using only infra-red light Prof. Wood has obtained photographs in which the sun shines, though there was only a grey cloudiness visible.

Though the colour sensitising of plates is now as much the manufacturer's business as the preparation of emulsions and the coating of the plates therewith, the experimentalist may wish to try the effect of a dye for himself. The solution must be very weak indeed, say one of the dye to from ro, ooo to 50,000 times its weight of water. Ammonia is often advantageous to the extent of one per cent. of the whole. The best formula is simply a nuatter of experiment. The following, for example, is a varation recently published by Messrs. Michaud and Tristan (Brit. Jnl. of Phot., lxii., 56) for sensitising for the red and infrared :-

Alcohol 5o per cent., 200 c.c.

Ammonia, 4 c.c.

Alizarin blue S, 0.04 gram.

Silver nitrate ro per cent. solution, 5 drops.

The same dye is efficient without the silver salt and the alcohol, but presumably these are advantageous. In general, after the plate has been in the dye solution for two or three minutes, it is washed for a few minutes and then dried. Plates so treated vary very much as to the time that they will remain in good condition. For example, plates bathed in the solution quoted above must be used within a few hours, but in other cases they may last for months.

$$
\text { NO. } 2365 \text {, VOL. 94] }
$$

It is much less risky to make colour filters than to bathe plates, as most dye solutions will be absorbed by a clean gelatine film. But colour filters are now made by several firms, and the Wratten department of Kodak catalogues standardised colour filters of nearly a hundred different transmissions.

Chapman Jones.

\section{PROF. G. F. J. ARTHUR AUWERS}

$\mathrm{TE}$ brief paragraph in our issue of last week, announcing the death of Prof. Auwers, must have been read with the deep regret that follows the loss of one who long occupied a commanding position in the world of science, and whose place it will be difficult to fill. For more than fifty years he had illuminated the science of astronomy, and by providing much of the material by which it is hoped to attack successfully the problem of the structure of the sidereal universe, he linked together the astronomical thoughts and methods of the past with the philosophical problems that engross and captivate the attention of astronomers of to-day.

In the history of astronomy of position, by which is understood the accumulation and arrangement of facts depending on a star's place in the sky, three names stand out prominently. Bradley, in whose valuable series of observations, long waiting for an interpreter, lay hidden the secret of stellar proper motion; Bessel, who made these measures available to the astronomers of his day; and Auwers, whose early appreciation of the necessity of the highest accuracy gave to these observations an increased value by his long and patient examination, laying. the foundation of that system of thorough uniformity which has welded meridian observations into a more consistent whole, facilitating the combination of star catalogued places on a common basis, by the removal of systematic errors or discrepancies. Auwers taught the necessity for a higher standard of accuracy, and it is not too much to say that in the department of reduction and discussion of observations he long stood without a rival. His forte lay in the control and management of large masses of work, in the unhurried, careful supervision of every stage, bringing an acute and trained judgment to bear eyually on all parts of the investigation.

If his reputation rests especially on his re-reduction of Bradley, it must be remembered that he encouraged and assisted other large undertakings. He tr - a prominent part in the re-observation of the Durchmusterung zones, a work of many years' international co-operation successfully carried out under the auspices of the Astronomische Gesellschaft. He was among the first to investigate the proper motion of faint stars, and he foreshadowed some of the conclusions that have been established by the most modern and thorough of inquiries. The determination of solar parallax by the method of the transit of Venus is a somewhat discredited problem now, but forty 